\title{
SEGUNDAS LENGUAS EN EL CURRICULUM NACIONAL CHILENO: TENSIONES HISTÓRICAS Y EPISTEMOLÓGICAS
}

\author{
SECOND LANGUAGES IN THE CHILEAN NATIONAL CURRICULUM: \\ HISTORICAL AND EPISTEMOLOGICAL TENSIONS.
}

\author{
María Francisca Lohaus-Reyes ${ }^{1}$ \\ Magister en Educación, mención Currículo y Comunidad Educativa \\ Universidad de Chile \\ mlohaus@ug.uchile.cl
}

\begin{abstract}
Resumen: El curriculum nacional se constituye como un lugar donde se selecciona qué información cultural se considera importante o imprescindible de ser transmitida a los habitantes de un país. Esta selección genera tensiones que se reflejan en el curriculum, en sus contenidos y -particularmente- en las lenguas que se enseñan. Este trabajo aborda algunas tensiones epistemológicas e históricas respecto a la enseñanza de segundas lenguas en el sistema educativo chileno y cómo la presencia o ausencia de ellas en el Curriculum Nacional da cuenta de las decisiones tomadas respecto a qué lenguas relevar y cuáles descartar en su selección cultural transmisible. Se advierte la promoción del bilingüismo angloamericano monocultural, así como diferencias en las fundamentaciones epistemológicas de las bases curriculares y de las asignaturas que se imparten, encontrando tensiones entre lo instrumental y global, lo holístico y local, la obligatoriedad y la optatividad.
\end{abstract}

Palabras clave: Curriculum Nacional, Segundas Lenguas, Lenguas ancestrales, Inglés como lengua extranjera, bilingüismo.

Resumo: 0 currículo nacional constitui-se como um local onde são selecionadas as informações culturais consideradas importantes ou essenciais para serem transmitidas aos habitantes de um país. Essa seleção gera tensões que se refletem no currículo, nos seus conteúdos e -particularmente- nas línguas que são ministradas. Este trabalho aborda algumas tensões epistemológicas e históricas a respeito do ensino de segundas línguas no sistema educacional chileno e como a presença ou ausência delas no Currículo Nacional explica as decisões tomadas sobre quais línguas pesquisar e quais rejeitar. sua seleção cultural transmissível. Nota-se a promoção do bilinguismo monocultural anglo-americano, bem como diferenças nos fundamentos epistemológicos das bases curriculares e das disciplinas ministradas, encontrando tensões entre o instrumental e o global, o holístico e o local, o obrigatório e o opcional.

\footnotetext{
${ }^{1}$ Núcleo de Estudio Curriculum, Conocimiento y Experiencia Educacional, Universidad de Chile
}

Fecha recepción: 10 de junio de 2021 
Palavras chave: Currículo nacional, segundas línguas, línguas ancestrais, inglês como língua estrangeira, bilinguismo.

Abstract: The national curriculum is constituted as a place where the cultural information that is considered important or essential to be transmitted to the inhabitants of a country is selected. This selection generates tensions that are reflected in the curriculum, in its contents and -particularly- in the languages taught. This paper addresses some epistemological and historical tensions regarding the teaching of second languages in the Chilean educational system and how the presence or absence of them in the National Curriculum accounts for the decisions made regarding which languages to emphasize and which to discard in its transmissible cultural selection. The promotion of Anglo-American monocultural bilingualism is noted, as well as differences in the epistemological foundations of the curricular bases and the subjects taught, finding tensions between the instrumental and global, the holistic and local, the compulsory and the electivity.

Key words: National Curriculum, Second Languages, Ancestral Languages, English as a foreign language, bilingualism.

En el territorio curricular se develan entramados complejos entre la globalización, las ideologías que sustentan los cambios culturales y económicos y las modificaciones exigidas al sistema educativo (Zajda, 2018), que tiene como misión formar a los futuros ciudadanos del país. El curriculum se sitúa como un espacio de creación y recreación constante de significados, donde se plasman tanto los sustentos teóricos, ideológicos y políticos que piensan la sociedad que se desea construir (Pinar, 2014), donde se selecciona qué información cultural se considera importante o imprescindible de ser transmitida. Es por ello que la educación institucionalizada, diseñada y orientada por la hoja de ruta trazada por el curriculum nacional, se convierte en una expresión de fuerzas e intereses diversos que culminan al materializarse en el acto educativo (Sánchez, 2001).

Las ideas expresadas en el diseño curricular distan de ser neutras o faltas de intenciones, intereses o sentido, sino que son afirmaciones cargadas de ideología y decisiones políticas y epistemológicas, que manifiestan abiertamente qué asignaturas deben ser relevadas, la forma de enseñarlas, el tiempo dedicado a ellas y qué medios se utilizarán para enseñarlas (como los libros de texto y las planificaciones sugeridas). Lo anteriormente mencionado transforma al currículum en un campo en constante tensión por luchas económicas, culturales, identitarias y corporativas (Apple, 2018). Estas tensiones se complejizan aún más dentro del paradigma global que considera el concepto de "sociedad del conocimiento" (Organización para la Cooperación y Desarrollo Económico, 2009). Esta declaración de existencia de una sociedad única y de un conocimiento único, permea el pensamiento que realiza las preguntas curriculares. Como consecuencia, diversos conocimientos y lenguas, que para el modelo economicista y neoliberal no poseen el valor suficiente para ser incluidos, se invisibilizan. Olivé (2009) afirma, en resistencia a lo anterior, una "sociedad de conocimientos", donde tanto los individuos como los colectivos pueden apropiarse y acceder a los conocimientos existentes -generados en cualquier lugar-, aprovechar el acervo experiencial, científico, tradicional y teórico acumulado durante el transcurso de la historia y generar, a su vez, nuevos conocimientos para comprender y 
resolver adecuadamente sus problemáticas. Parte de los desafíos que enfrenta el diseño curricular es situarse de forma crítica ante una realidad que parece ser única, pero es sólo una parte de un espectro múltiple, donde existen cruces epistemológicos, lingüísticos y culturales que deberían ser explorados y considerados.

Este proceso dialéctico entre currículum y sociedad genera tensiones que, lejos de resolverse, se reflejan en el currículum, en sus contenidos y -particularmente- en las segundas lenguas que se enseñan, así como la obligatoriedad u optatividad de estas, transformándose el curriculum en un "campo de batalla", como afirma Apple (2018). A medida que la globalización ha abierto nuevos canales y espacios para la comunicación, que según Süselbeck y Mühlschlegel (2008) hacen necesaria una reducción de la diversidad lingüística, algunas políticas locales y las minorías lingüísticas han llevado a cabo un trabajo de promoción y relevancia de las lenguas menos difundidas. Estos territorios lingüísticos entrecruzados se reconocen, por lo tanto, como territorios en disputa por el poder y por la identidad (Süselbeck y Mühlschlegel, 2008; Cummins, 2002).

Para efectos de este análisis, se situará esta discusión de la presencia o ausencia de las segundas lenguas en el curriculum en su contexto histórico, debido a que el campo curricular existe y se encarna bajo las subjetividades que habitan en él, además de dialogar con los conocimientos que atraviesan lo prescrito, lo enseñado, lo aprendido y lo vivido en ese momento de la historia (Pinar, 2014). Las lenguas que "habla" (o silencia) el currículum chileno da cuenta de las decisiones que la sociedad ha tomado, respecto a qué lenguas destacar y cuáles descartar al momento de realizar la selección cultural que ha de ser transmitida a los habitantes de la nación.

\section{TENSIONES HISTÓRICAS}

Desde el inicio de la República, se dio relevancia a la enseñanza de segundas lenguas. Se puede observar que en el primer plan de estudios del Instituto Nacional (la primera escuela fundada en el proceso de emancipación), aparecían lenguas como el francés, el latín y el inglés (Campos, 1965). Al fundarse la Universidad de Chile, se tenía en mente formar profesores de idiomas extranjeros (Vivanco, 2016). Andrés Bello, el primer rector, consideraba que el estudio de esos idiomas era un medio de "comunicación con la antigüedad y con las naciones más civilizadas, cultas y libres de nuestros días" (Bello, en Vivanco, 2016). La élite intelectual compartía estos ideales, ya que la república naciente debía acercarse al ideario de la ilustración europea. Mignolo (2006) afirma que las naciones transitaron a la modernidad a través de la enseñanza y difusión de lenguas derivadas del griego y del latín. En el siglo XIX, Chile consolidó y centralizó su sistema educativo con un aparataje que incluyó la fundación del Ministerio de Educación (Antoine, 2007; Campos, 1965). Las reformas estructurales desembocaron en mayor regulación administrativa y, por consiguiente, de los planes y programas de estudio.

Con el paso de las décadas, los planes de estudio prescribirían las lenguas extranjeras como electivas. En el año 1875, se realiza una segunda reforma, que las decreta como obligatorias durante los primeros cuatro años de las humanidades; una tercera reforma 
(1889) restringió la enseñanza de lenguas extranjeras (inglés y alemán) sólo al quinto y sexto año de humanidades. Después del cambio de siglo, las lenguas extranjeras volvieron a tomar carácter obligatorio (Campos, 1965).

El gobierno de Eduardo Frei Montalva llevó a cabo la reforma educacional de 1965, con transformaciones significativas para todo el sistema educativo. Dentro de las consideraciones curriculares, en los planes de estudio de Enseñanza Media HumanísticoCientífica se decretó la enseñanza de un idioma extranjero (francés o inglés), con carácter optativo (Núñez, 2003). El mismo autor (2003) señala que durante la década de los 70, la administración de Allende continuó con el trabajo curricular realizado en el gobierno anterior.

La irrupción de la dictadura cívico-militar impuso diversas medidas de reestructuración interna del Ministerio de Educación, que tuvieron lugar en la década de los 80 y que afectaron al sistema escolar tanto en lo administrativo como en lo curricular. A través del decreto №4002 se implementa la enseñanza optativa en quinto y sexto básico de dos idiomas extranjeros (entre los cuales se podía elegir entre inglés, alemán y francés), sujeta a la disponibilidad de docentes por parte del establecimiento, mientras que en séptimo y en octavo básico la enseñanza de esas lenguas sería obligatoria. El director y la jefatura técnica estaban a cargo de escoger las segundas lenguas a enseñar, realizando una consulta previa al centro de padres de la escuela o liceo (Decreto №4002, art.15, 1980).

En cuanto a los objetivos planteados para las asignaturas de alemán o francés en la educación básica y media, estaba incluida la interacción en diálogos simples de la vida cotidiana, lectura comprensiva de textos sencillos y la apreciación de aspectos culturales de los países que hablaran dichas lenguas (Decreto $\mathrm{N}^{\circ}$. 4002, 1980, art. 23). Por otra parte, los objetivos planteados para el idioma extranjero inglés fueron ampliados, incluyendo además la escucha de canciones, observación de películas, acceso a literatura general y uso del idioma en actividades de esparcimiento (Decreto $\mathrm{N}^{\circ} 4002$, 1980, art.23; Decreto exento $\mathrm{N}^{\circ} 300$, 1982). En el año 1984, la obligatoriedad de enseñanza de idiomas extranjeros fue reducida a una lengua, decisión que influyó en que la opción curricular mayoritaria de las escuelas y liceos fuese el inglés (Decreto exento 3, 1984; Antoine, 2007). Con esta medida se constriñó el espacio curricular que habían ocupado las otras lenguas en el currículum.

Los gobiernos de transición, posteriores a la dictadura, quisieron marcar la diferencia con el régimen predecesor, implementando cambios profundos al sistema educativo. Dentro de las reformas de los años 90, se declararon fines estratégicos, como la mejora de los resultados educativos en las mediciones estandarizadas y la actualización de las competencias de las personas que egresaban de la educación obligatoria, para permitir una mejor incorporación de ellas al mercado laboral (Donoso, 2004). Con ese fin, se realizaron cambios en los planes y programas, además de modificaciones estructurales. Entre ellas, la institución de la Jornada Escolar Completa y la reducción de la oferta de segundas lenguas a sólo una, de carácter obligatorio (Decreto $\mathrm{N}^{\circ}$ 240, 1999). Según Antoine (2007), en la normativa no se explicitó ninguna lengua en particular, pero el Ministerio de Educación realizó diversas campañas para promover el inglés, a través de cursos gratuitos para estudiantes y becas de perfeccionamiento para los profesores, además de facilitar la 
conversión de profesores de otras lenguas para que se especializaran en la enseñanza del inglés (MINEDUC, 2004). Ante el despliegue demostrado por la oficialidad, los establecimientos adoptaron la propuesta del MINEDUC, restringiendo la oferta lingüística al inglés. Tal preferencia queda aún más de manifiesto con la profundización de las medidas ya impulsadas para promover la enseñanza y el aprendizaje del inglés, traducidas en su mayoría por el Programa Inglés Abre Puertas (MINEDUC, 2004; Glas, 2008).

\section{Contexto legal}

En Chile no existe una lengua oficial por ley, sólo existe una lengua de facto, que es el español. En la constitución política vigente, tampoco existe un reconocimiento, protección o relevancia de la lengua mayoritaria (Antoine, 2007). La derogada Ley Orgánica Constitucional de Enseñanza (LOCE), exigía la adquisición y valorización del lenguaje escrito y oral (Ley 18.962, art.13, 1990), pero no menciona en qué lengua debía lograrse dicho objetivo. En la posterior Ley General de Educación (LGE), se establece, como parte de los objetivos generales dictados para el egreso de la educación media: "Expresarse en lengua castellana en forma clara y eficaz, de modo oral y escrito; leer comprensiva y críticamente diversos textos de diferente nivel de complejidad (...)y tomar conciencia del poder del lenguaje para construir significados e interactuar con otros."(Ley 20.370, 2009, Art. 30, numeral 2, letra d) y "comprender el lenguaje oral y escrito de uno o más idiomas extranjeros, y expresarse en forma adecuada." (Ley 20.370, 2009, Art. 30, numeral 2, letra f). Se puede apreciar que hay un paso hacia la valoración de la lengua mayoritaria del país, pero, por otra parte, se realiza una distinción entre esta y las segundas lenguas (y lenguas extranjeras), en cuanto al grado de profundidad de los objetivos de aprendizaje planteados para cada una de ellas.

El Ministerio de Educación declara a Chile como un país multicultural y plurilingüe en las bases curriculares (2009), desafiando a los establecimientos educacionales a transformarse en espacios de aprendizaje accesibles a las infancias de diversas culturas y lenguas, de forma pertinente a su edad y sistemáticamente. Junto con esta asunción, se ha creado un espacio curricular de una asignatura para las lenguas de los pueblos originarios, traduciéndose en programas de Educación Intercultural Bilingüe (EIB) en lengua Rapa Nui, Aymara, Quechua y Mapudungun y Rapa Nui -el Sector Lengua Indígena (SLI, Decreto 280/2009) - en la que se incorporan unidades programáticas que permitan a los y las estudiantes abordar aspectos de su lengua y cultura en la que viven (Ley 19.253, 1993). El SLI entró en vigencia gradualmente, desde el año 2010 (MINEDUC, 2017; 2017b; 2017c; 2017d). El decreto 280 señala que el SLI debe ofrecerse obligatoriamente en los establecimientos educacionales con matrícula indígena igual o mayor al $50 \%$, mientras que la incorporación de esa asignatura sería opcional en el caso de los establecimientos con una matrícula indígena menor al 20\%. La construcción de las bases curriculares de Lengua Indígena consideró la consulta (no vinculante) a profesores de establecimientos con matrícula indígena significativa, especialistas, comunidades, hablantes de las lenguas originarias y organizaciones (MINEDUC, 2017; 2017b; 2017c; 2017d). El objetivo de esa asignatura es lograr que los estudiantes usen la lengua como mecanismo de comunicación 
intercultural e interacción, que reproduzcan discursos y textos relacionados con eventos y ceremonias propios de su comunidad y cultura y como medio de resolución de problemas (MINEDUC, 2017; 2017b; 2017c; 2017d).

Es importante destacar que los cinco pueblos restantes reconocidos por ley Licanantai, Colla, Diaguita, Kawésqar, Yagán y Chango- tienen la posibilidad de realizar talleres de revitalización en los establecimientos educacionales, pero se trata de espacios que no garantizan la continuidad de la experiencia formativa, ya que, hasta el momento, dependen de proyectos anuales, en contraste con el aparataje estatal y económico que presenta la enseñanza del inglés como lengua extranjera. Se espera que para el 2021 se publiquen las bases curriculares de Lengua y Cultura de los Pueblos Originarios Ancestrales para los nueve pueblos.

A lo anterior se suma el reconocimiento de la Lengua de Señas chilena como medio natural de comunicación de la comunidad sorda en el país, en la ley de igualdad de oportunidades e inclusión de personas con discapacidad (Ley 20.422, 2010) y la aprobación de los planes y programas de enseñanza media MINEDUC de un Colegio que imparte Educación Intercultural Bilingüe en Lengua de Señas Chilena (Colegio Dr. Jorge Otte Gabler, 2020). Recientemente fue aprobada la ley 21.303 (2021), que modifica el apartado de la Lengua de Señas chilena, reconociéndola como "lengua natural, originaria y patrimonio intangible de las personas sordas", además de mencionar su rol esencial como configuradora de la cultura de la comunidad sorda. Desde la perspectiva legal, se podría afirmar que se reconoce una diversidad lingüística en el país, pero que no se configura como una característica identitaria dentro de la base cultural común de la nación.

\section{TENSIONES EPISTÉMICAS: GLOBAL-LOCAL, MULTICULTURALIDAD, INTERCULTURALIDAD Y PLURILINGÜISMO}

La sociedad chilena se declara, a través de su curriculum, como multicultural y plurilingüe (MINEDUC, 2009). Se puede apreciar, ya desde esta declaración de principios, que los fundamentos de ambas definiciones son completamente opuestos. En este contexto de reconocimiento y legitimación de las diversas lenguas en el curriculum, se posicionan tres conceptos clave: multiculturalidad, interculturalidad y plurilingüismo.

La definición de multiculturalidad se puede abordar como un rasgo correspondiente a los países, en tanto territorios compuestos por múltiples culturas y etnias (Sirlopú, Melipillán, Sánchez y Valdés, 2015) o como apertura a la heterogeneidad cultural desde lo político, que comienza en la convivencia armónica, mutua, horizontal, situada y respetuosa de las diferencias (Stefoni, Stang y Riedemann, 2016). Zizek (2003) sostiene que la multiculturalidad es un producto de la auto colonización de los pueblos, donde las culturas originarias son "nativas" que deben "respetarse", en una suerte de desdén epistemológico y condescendencia. Tal "distancia respetuosa" se transforma en un espacio vacío en el que no hay oportunidad de encuentro legítimo, sino un ejercicio de superioridad autoimpuesta (Mignolo, 2006), que se consolida en la observación y reconocimiento -lejano- del otro "diferente". La multiculturalidad, desde la mirada crítica de Zizek, es una concepción de 
culturas inamovibles, ajenas a las tensiones y movimientos que se generan entre ellas y que desconoce la falta de horizontalidad de sus relaciones, debido a los procesos históricos y sociales que les dan origen. El concepto de "mirar desde lejos" o de "respetuosa distancia" también puede encontrarse en los contextos de enseñanza y aprendizaje de las lenguas extranjeras, en tanto se limita a una comprensión superficial de la lengua extranjera desde el sitial autoimpuesto de la propia cultura, donde se interpreta lo que se ve, pero no hay un esfuerzo de averiguar si la forma de pensar que se está enseñando es válida para explicar comportamientos lingüísticos o actitudes del otro (Iglesias, 1998). El autor se refiere a este fenómeno como el "efecto escaparate".

Por otra parte, sólo la interculturalidad puede dar a luz al plurilingüismo. Este debe provenir de un intercambio democrático, legítimo y real de conocimientos entre dos o más culturas que se lleva a cabo entre los individuos (Fornet-Betancourt, 2002). Es un proceso intencional, horizontal e igualitario que dos o más culturas hacen de incorporar la diversidad en sus propias culturas y deconstruir, compartir, modificar y transferir lo necesario en ellas y entre ellas (Stefoni et al., 2016). La interculturalidad cruza los límites de la tolerancia y comprende el intercambio horizontal y dialógico de saberes como un proceso vivo y consciente, que va en aras del enriquecimiento mutuo entre dos culturas y configura la convivencia de las diferencias, para generar cambios tanto prácticos como teóricos (TrujilloSáez, 2008; Fornet-Betancourt, 2002). En este sentido, se valoran las diversidades como algo deseable en vez de un problema. Según Cummins (2006), la tarea de comprender los procesos de interculturalidad está vinculada estrechamente con comprender las relaciones de poder y cómo estas relaciones determinan las expectativas de aprendizaje, las dinámicas de enseñanza y aprendizaje en el aula, los objetivos propuestos y las decisiones curriculares en todos los niveles. Asumir esta posición epistémica implica entablar un diálogo consciente que permita que los saberes de cada diversidad puedan encontrarse y aportar la riqueza que poseen (Lohaus-Reyes, 2019).

La interculturalidad puede plantearse, según Iglesias (1998) como una trayectoria de aprendizaje mutuo, donde ninguna cultura parte desde cero y ocurren comparaciones y descodificaciones que parten desde los saberes y experiencias previas, pero donde también ocurren los cruces entre conocimientos propios de cada cultura, que son una fuente de riqueza indiscutible. Es en este espacio de cruces y aprendizajes donde se encuentra el desafío de generar propuestas curriculares que no planteen los saberes como entidades inamovibles y cerradas, sino más bien como formas diferentes de vivir, pensar e interpretar el mundo (Stefoni et. al, 2016). La legitimación y el reconocimiento mutuos conlleva la valorización de la pluralidad lingüística y cultural.

Las posibilidades de enseñanza y aprendizaje en el paradigma interculturalplurilingüe pueden llevar a una mayor comprensión de cómo se construyen las lenguas y a tomar las estrategias bilingües de los estudiantes como fortalezas en el proceso de aprendizaje de una segunda, tercera o cuarta lengua (Piccardo, 2013).

A continuación, se observará cómo las diferencias epistémicas (planteadas ya desde las bases curriculares y el marco legal) permean el currículum de segundas lenguas y lenguas 
extranjeras, debido a que se piensan en visiones completamente dispares e incompatibles entre sí.

En las bases curriculares de lengua indígena la lengua es un conocimiento fundamental en el proceso de valoración y comprensión de la cultura por parte del estudiante, por lo que el aprendizaje de la lengua va unido al aprendizaje de la relación con la naturaleza y la posición del ser humano en ella, las cosmovisiones de cada cultura y la convivencia y armonía entre los pares y la comunidad (MINEDUC, 2009). Por otra parte, las bases curriculares de inglés como lengua extranjera (MINEDUC, 2015c) están centradas en la extensión mundial de su uso en ámbitos como la economía, tecnología, ciencia y academia y cómo Chile se ha incorporado en el proceso de globalización. Se considera el aprendizaje del inglés, por lo tanto, como importante en la participación colectiva e individual en las dinámicas globales, debido a los beneficios que traen a la sociedad. Para ello, el idioma inglés se configura en el curriculum nacional como una herramienta de acceso a la información para las y los estudiantes, como una forma de apreciar formas de pensamiento, tradiciones y estilo de vida distintos y desarrollar habilidades cognitivas de orden superior (MINEDUC, 2009).

En las bases curriculares de lengua indígena, existe un reconocimiento de que los niños bilingües tienen dos formas de representar la realidad, con lo cual pueden pensarla y escribirla de distintas maneras, tienen mayor capacidad de adaptarse a los cambios y comprender creativamente el entorno y mundo en que viven (MINEDUC, 2009). Las bases curriculares, los contenidos mínimos obligatorios y los objetivos fundamentales destacan la importancia del bilingüismo inglés para que, al egreso de la enseñanza obligatoria, las personas puedan participar del mundo laboral y la educación superior, las comunicaciones, tecnología, ciencia y comercio (MINEDUC, 2009; 2013).

Se puede apreciar una diferencia clave en las fundamentaciones epistemológicas del currículum de segundas lenguas, extranjera e indígena: el enfoque de la asignatura de inglés tiene un carácter claramente instrumental y está planteado desde una perspectiva subyugada a lo global y económico que, a lo local y holístico, que es el caso del currículum de lenguas originarias. Se advierte una promoción del monolingüismo angloamericano como sinónimo de modernidad, ciencia y éxito económico (Antoine, 2007) en lugar de situar las lenguas en el currículum en igualdad de condiciones. El discurso público del gobierno promovía expresamente el inglés como una manera de "garantizar el éxito económico del país" (Glas, 2008), soslayando las ventajas sociales y cognitivas que posee aprender una segunda lengua. Posteriormente, las bases curriculares incorporaron la importancia del aprendizaje de otras lenguas como formas de promoción de la diversidad lingüístico-cultural y de respetar formas distintas de actuar en el mundo (MINEDUC, 2015c; 2017, 2017b; 2017c; 2017d). JohnsonMardones (2015) plantea que en el discurso curricular-tecnocrático se celebra la diversidad como parte de lo global, pero a la vez dicha diversidad se ve subsumida dentro de un proceso de homogeneización. En este sentido, es urgente la articulación de las diversas lógicas que habitan en el espacio curricular, para discutir las posiciones hegemónicas y proponer alternativas al modelo monocultural y anglo-bilingüe propuesto por el curriculum centralizado (Quilaqueo et.al, 2014). La reducción de las lenguas a un mero instrumento comunicativo (como lingua franca, vehículo de la globalización y el comercio) y a los estudiantes como engranajes de una máquina de producción (Vivanco, 2016) es un atentado 
contra la riqueza cultural y educativa que poseen las lenguas, como también al valor de la educación como fin en sí mismo.

\section{REFLEXIONES FINALES}

El primer desafío que se vislumbra ante lo expuesto es la revisión de la selección cultural (y en este caso, lingüística) presente en el curriculum nacional. ¿Qué lenguas se legitiman y cuáles no y por qué? ¿Cuáles son las concepciones curriculares que permean las metodologías de enseñanza, la formación de educadores y profesores? ¿Qué lenguas deberíamos dominar al finalizar nuestro recorrido por la enseñanza obligatoria? ¿Será el momento de ampliar la oferta e ir más allá del bilingüismo angloamericano que los diversos gobiernos han tratado de promover?

Es importante mantener la vigilancia epistémica en el momento de la configuración global de las asignaturas que conforman el curriculum nacional. Esto quiere decir que las lenguas no son sólo medios de comunicación o herramientas útiles para fines de productividad, sino que son formas de construcción y expresión de realidades, formas de concebir cosmovisiones y son la base desde donde se erigen las formas de hacer ciencia y de dialogar con otras posturas y culturas. En lo práctico, los pueblos originarios deben ser partícipes no sólo de un sector o asignatura del curriculum nacional, sino que deben colaborar y estar presentes en el diseño curricular global (Inostroza-Barahona \& LohausReyes, 2019), más allá de procesos de consulta (que, al ser no vinculantes, menoscaban su participación), ya que las lenguas permean todos los ámbitos de los diferentes saberes que conviven en el territorio nacional, más aún si se desea relevar las lenguas ancestrales. Se requiere generar y articular las diversas lógicas de conocimiento, para discutir las hegemonías presentes en el curriculum nacional e incorporar los saberes educativos de los pueblos originarios, para avanzar desde la monoculturalidad hacia la interculturalidad, de un monólogo curricular a una conversación curricular (Johnson-Mardones, 2020).

En la misma línea, es imperativo que exista una valoración lingüística de las lenguas que se hablan (o señan) desde el marco legal, principalmente en la constitución. No basta con asumir que en Chile se habla/escribe español porque así ha sido por varios siglos. Como dice un viejo refrán: "por sabido se calla, por callado se olvida". No es un punto menor, considerando que el dominio de una lengua (mayoritaria o materna) pavimenta el camino para los aprendizajes posteriores y que las lenguas son parte importante en la tan anhelada configuración de una identidad nacional. Claramente, una ley no hace praxis, pero sí permite que las políticas públicas respecto a la enseñanza de las diversas lenguas del CN puedan adecuarse y potenciarse de forma horizontal.

Contrasta también la poca relevancia entregada a la formación de Profesores y Educadores en cuanto a fortalecer las capacidades pedagógicas de su lengua, en especial si se piensa que la asignatura de Lengua y Cultura de los Pueblos Originarios Ancestrales se considera actualmente como una asignatura abierta para cualquier escuela o liceo que desee incorporarla. ¿Cuáles podrían ser las metodologías para enseñar una lengua de pueblo originario a una persona que nunca la ha hablado, que no tiene contacto directo ni constante 
con ella ni con la tradición que le da origen? Es imperativo fortalecer una institucionalidad que promueva formación inicial docente acorde a los objetivos que se pretenden para las nuevas asignaturas, más aun considerando que se impartirán durante los trece años de enseñanza obligatoria.

El ideal sería que el curriculum transitara hacia una concepción dinámica, abierta y pluralista del bilingüismo, la enseñanza y del aprendizaje de las segundas lenguas, como coadyuvantes en el logro cognitivo, cultural y comunicativo de los y las estudiantes, situando las lenguas locales como el lugar donde se aprecien y abracen todas las culturas que hay por conocer, más allá de intereses hegemónicos, colonizantes y y utilitarios. 


\section{REFERENCIAS BIBLIOGRÁFICAS}

Antoine, M. N. (2007). El francés en el sistema educativo chileno: causa perdida o caballo de troya para un cambio. Contextos, estudios de humanidades y ciencias sociales, 18, 203-210.

http://revistas.umce.cl/index.php/contextos/article/viewFile/499/495

Apple, M. W. (2018). ¿Puede la educación cambiar la sociedad?. LOM ediciones.

Campos, F. (1965). Desarrollo educacional 1810-1960. Santiago, Chile: Andrés Bello.

Colegio Dr. Jorge Otte Gabler (2020). Proyecto educativo institucional intercultural bilingüe. https://institutodelasordera.cl/documentos/1.\%20PEI\%202020.pdf

Cummins, J. (2002). Lenguaje, poder y pedagogía: niños y niñas bilingües entre dos fuegos. Madrid, España: Ediciones Morata.

Decreto $\mathrm{N}^{\circ}$ 4002. Fija objetivos, planes y programas de la Educación General Básica. Santiago, Chile, 5 de junio de 1980. http://bcn.cl/2kqfo

Decreto $\mathrm{N}^{\circ} 300$ Exento. Aprueba planes y programas para la Educación Media Humanístico Científica. 22 de Enero de 1982. https://legislacion-oficial.vlex.cl/vid/planeseducacion-humanistico-cientifica-497595582\#: :text=Decreto\%20n\%C3\%BAm.300\%20EXENTO\%2C\%20publicado\%20el\%2022\%20de\%20Enero\%20de $\% 20198$ 2.,LA\%20EDUCACION\%20MEDIA\%20HUMANISTICO\%2DCIENTIFICA

Decreto 3 exento. Modifica decreto supremo exento $N^{\circ} 300$, de 1981. 30 de Enero de 1984.

Decreto $\mathrm{N}^{\circ}$ 240. Modifica decreto № 40, de 1996. 16 de junio de 1999.

Donoso, S. (2004). Reforma y Política Educacional en Chile 1990-2004: el neoliberalismo en crisis. Estudios Pedagógicos, 31(1), 113-135. http://www.opech.cl/bibliografico/Doc Financiamiento/Reforma Educativa El Neo liberalismo En Crisis Donoso.pdf

Fornet-Betancourt, R. (2002). Lo intercultural: el problema de su definición. Pasos, 103,1-3.

Iglesias, I. (1998). Diversidad cultural en el aula de E/LE: la interculturalidad como desafío y como provocación. En El español como lengua extranjera: del pasado al futuro: actas del VIII Congreso Internacional de ASELE, (Alcalá de Henares, 17-20 de septiembre de 1997) (pp. 463-472). https://dialnet.unirioja.es/descarga/articulo/893231.pdf

Inostroza-Barahona, C. \& Lohaus-Reyes, M. F. (2019). Inclusión y diversidad: Nuevos desafíos para la política curricular chilena. Reflexiones desde la teoría curricular y la justicia social. Revista Internacional de Educación para la Justicia Social, 8(1), 151-162. https://doi.org/10.15366/riejs2019.8.1.009 
Johnson-Mardones, D. (2020) Estudos curriculares como conversação internacional: notas do sul. Revista Espaço do Curriculo, 13(3), 345-352. https://doi.org/10.22478/ufpb.1983-1579.2020v13n3.55434

Johnson-Mardones, D. (2015). Formar ciudadanos interculturales en un mundo global: algunas notas desde los estudios curriculares. Diálogo andino, (47), 7-14. https://dx.doi.org/10.4067/S0719-26812015000200002

Ley N 18.962 (derogada). Ley Orgánica Constitucional de Enseñanza. Diario Oficial de la República de Chile, 10 de marzo de 1990. http://www.uchile.cl/portal/presentacion/normativa-y-reglamentos/8386/leyorganica-constitucional-de-ensenanza

Ley $\mathrm{N}^{\circ}$ 21.303. Modifica la ley $N^{\circ}$ 20.422, que establece normas sobre igualdad de oportunidades e inclusión social de personas con discapacidad, para promover el uso de la Lengua de Señas. Diario Oficial de la República de Chile, Santiago, Chile. 22 de enero de 2021. http://bcn.cl/2nw2z

Ley $\mathrm{N}^{\circ}$ 20.422. Establece normas sobre igualdad de oportunidades e inclusión social de personas con discapacidad. Diario Oficial de la República de Chile, Santiago, Chile. 10 de febrero de 2010. http://bcn.cl/1uvqg

Ley $N^{\circ}$ 20.370. Ley General de Educación. Diario oficial de la República de Chile, 12 de septiembre de 2009, Santiago, Chile. https://www.leychile.cl/Navegar?idNorma=1006043

Lohaus-Reyes, M. F. (2019). Curricular Decolonization from Epistemologies of Diversity. Transnational Curriculum Inquiry, 16(1), 74-80.

Mignolo, W. (2006). La opción descolonial: el pachakuti conceptual de nuestro tiempo. Conceptos y fenómenos fundamentales de nuestro tiempo, Universidad Nacional Autónoma de México, Instituto de Investigaciones Sociales, 10.

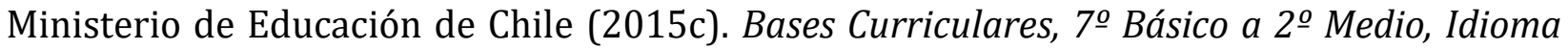
Extranjero inglés. http://www.curriculumenlineamineduc.cl/605/articles$\underline{34957 \text { Bases.pdf }}$

Ministerio de Educación de Chile (2009). Currículum. Objetivos Fundamentales y Contenidos Mínimos Obligatorios de la Educación Básica y Media. http://www.curriculumnacional.cl/614/articles-34641 bases.pdf

Ministerio de Educación de Chile (2004). Decreto 81, crea Programa Inglés Abre Puertas. http://ingles.mineduc.cl/pdf/dec 81.pdf

Ministerio de Educación de Chile (2017). Programa de estudio $7^{\circ}$ año básico, Sector Lengua Indígena Quechua_Ministerio de Educación: Gobierno de Chile. 
Ministerio de Educación de Chile (2017b). Programa de estudio $7^{\circ}$ año básico, Sector Lengua Indígena Rapa Nui_.Ministerio de Educación: Gobierno de Chile.

Ministerio de Educación de Chile (2017c). Programa de estudio $7^{\circ}$ año básico, Sector Lengua Indígena Mapuzungun_.Ministerio de Educación: Gobierno de Chile.

Ministerio de Educación de Chile (2017d). Programa de estudio $7^{\circ}$ año básico, Sector Lengua Indígena Aymara. Ministerio de Educación: Gobierno de Chile.

Núñez, I. (2003). La ENU entre dos siglos: ensayo histórico sobre la Escuela Nacional Unificada. Santiago, Chile: Lom Ediciones.

Olivé, L. (2009). Por una auténtica interculturalidad basada en el reconocimiento de la pluralidad epistemológica. VV.AA en "Pluralismo Epistemológico". Buenos Aires, Argentina: Clacso.

Organización para la Cooperación y Desarrollo Económico (2009). Learning for Jobs: OECD Reviews of Vocational Education and Training. http://www.oecd.org/education/skills-beyondschool/Learning\%20for\%20Jobs\%20book.pdf

Piccardo, E. (2013). Plurilingualism and curriculum design: Toward a synergic vision. TESOL Quarterly, 47(3), 600-614. DOI:10.1002/tesq.110

Pinar, W. F. (2014). La teoría del curriculum. Madrid, España: Narcea Ediciones.

Quilaqueo, D., Quintriqueo, S., Torres, H., \& Muñoz, G. (2014). Saberes educativos mapuches: aportes epistémicos para un enfoque de educación intercultural. Chungará (Arica), 46(2), 271-284. https://dx.doi.org/10.4067/S0717-73562014000200008

Sánchez, M. (2001). Diseño curricular y educación intercultural bilingüe. Pensamiento educativo, 29, 341-358.

Stefoni, C., Stang, F., \& Riedemann, A. (2016). Educación e interculturalidad en Chile: Un marco para el análisis. Estudios internacionales (Santiago), 48(185), 153-182. https://dx.doi.org/10.5354/0719-3769.2016.44534

Süselbeck, K., \& Mühlschlegel, U. (2008). Lengua, nación e identidad: la regulación del plurilingüismo en España y América Latina. Madrid, España: Iberoamericana Editorial.

Trujillo-Sáez, F. (2008). Plurilingüismo en el aula: las lenguas de los estudiantes. I Jornadas sobre Lenguas, Currículo y Alumnado Inmigrante. 61-70. http://segundaslenguaseinmigracion.com/Actas Congresos/Ijornadasdeusto .pdf\#page $=61$ 
Vivanco, H. (2016). Panorama histórico sobre la enseñanza de lenguas extranjeras en Chile. Lenguas Modernas, https://revistahistoriaindigena.uchile.cl/index.php/LM/article/view/45187

Zajda, J. (2018). Globalisation and education reforms: Paradigms and ideologies. En Globalisation and Education Reforms (pp. 1-14). Dordrecht, Holanda: Springer. 